\title{
MAFLD: A consensus-driven proposed nomenclature for metabolic associated fatty liver disease
}

\author{
Mohammed Eslam¹, Arun J. Sanyal², Jacob George ${ }^{1}$, on behalf of an international \\ consensus panel
}

\author{
${ }^{1}$ Storr Liver Centre, Westmead Institute for Medical Research, Westmead Hospital and \\ University of Sydney, NSW, Australia \\ ${ }^{2}$ Virginia Commonwealth University School of Medicine, Richmond, VA, 23284.
}

\section{Corresponding Authors:}

Mohammed Eslam: Storr Liver Centre, Westmead Institute for Medical Research, Westmead Hospital and University of Sydney, Westmead 2145, NSW, Australia, Ph: +61-2-88907705; Fx: +61-2-96357582. Email: mohammed.eslam@sydney.edu.au

Arun J. Sanyal: Virginia Commonwealth University School of Medicine, MCV Box 980341, Richmond, VA 23298-0341, United States. Tel.: +1 (804) 828 6314; fax: +1 (804) 8282992. E-mail address: arun.sanyal@vcuhealth.org

Jacob George: Storr Liver Centre, Westmead Institute for Medical Research, Westmead Hospital and University of Sydney, Westmead 2145, NSW, Australia, Ph: +61-2-88907705; Fx: +61-2-96357582. Email: jacob.george@ sydney.edu.au

Competing interest's statement: Rajiv Jalan has research collaborations with Takeda, and Yaqrit, and consults for Akaza and Yaqrit. Rajiv Jalan is the founder of Yaqrit Limited, which is developing UCL inventions for treatment of patients with cirrhosis. Rajiv Jalan is an inventor of ornithine phenylacetate, which was licensed by UCL to Mallinckrodt. He is also the inventor of Yaq-001, DIALIVE and Yaq-005, the patents for which have been licensed by his University into a UCL spinout company, Yaqrit Ltd. The rest of authors declare no competing interests for this manuscript.

Acknowledgements: ME and JG are supported by the Robert W. Storr Bequest to the Sydney Medical Foundation, University of Sydney; a National Health and Medical Research Council of Australia (NHMRC) Program Grant (APP1053206, APP1149976) and Project grants (APP1107178 and APP1108422). PNN is funded and supported by the National Institute for Health Research (NIHR) Birmingham Biomedical Research Centre at the University Hospitals Birmingham NHS Foundation Trust and the University of Birmingham. The views expressed are those of the authors and not necessarily those of the NIHR, the Department of Health and Social Care or the NHS. 


\section{The International Consensus Panel (in alphabetical order)}

Arun Sanyal, Virginia Commonwealth University School of Medicine, Richmond, VA, USA.

Brent Neuschwander-Tetri, Division of Gastroenterology and Hepatology, Saint Louis University, St. Louis MO, USA.

Claudio Tiribelli, Liver Center, Italian Liver Foundation, Trieste, Italy.

David E. Kleiner, Laboratory of Pathology, Center for Cancer Research, National Cancer Institute, Bethesda MD, USA

Elizabeth Brunt, Department of Pathology and Immunology Washington University School of Medicine St Louis MO, USA.

Elisabetta Bugianesi, Division of Gastroenterology and Hepatology, Department of Medical Sciences, University of Turin, Turin, Italy.

Hannele Yki-Järvinen, Department of Medicine, University of Helsinki and Helsinki University Hospital, and Minerva Foundation Institute for Medical Research, Helsinki, Finland.

Henning Grønbæk, Department of Hepatology \& Gastroenterology, Aarhus University Hospital, Aarhus, Denmark.

Helena Cortez-Pinto, Clínica Universitária de Gastrenterologia, Laboratório de Nutrição, Faculdade de Medicina, Universidade de Lisboa, Portugal.

Jacob George, Storr Liver Centre, Westmead Institute for Medical Research, Westmead Hospital and University of Sydney, NSW, Australia

Jiangao Fan, Center for Fatty Liver, Department of Gastroenterology, Xin Hua Hospital Affiliated to Shanghai Jiao Tong University School of Medicine, Shanghai, China.

Luca Valenti, Department of Pathophysiology and Transplantation, Università degli Studi di Milano, and Translational Medicine, Department of Transfusion Medicine and Hematology, Fondazione IRCCS Ca' Granda Ospedale Maggiore Policlinico, Milano, Italy.

Manal Abdelmalek, Department of Medicine, Duke University, Durham, NC, USA.

Manuel Romero-Gomez, Hospital Universitario Virgen del Rocío de Sevilla, Instituto de Biomedicina de Sevilla, Biomedical Research Networking Center in Hepatic and Digestive Diseases, Sevilla, Spain.

Mary Rinella, Department of Gastroenterology, Northwestern University Feinberg School of Medicine, Chicago, IL, USA.

Marco Arrese, Departamento de Gastroenterología, Escuela de Medicina, Pontificia Universidad Católica de Chile, Santiago, Chile.

Mohammed Eslam, Storr Liver Centre, Westmead Institute for Medical Research, Westmead Hospital and University of Sydney, NSW, Australia 
Pierre Bedossa, Department of Pathology, Physiology and Imaging, Beaujon Hospital Paris Diderot University, Paris, France.

Philip N Newsome, National Institute for Health Research Biomedical Research Centre at University Hospitals Birmingham NHS Foundation Trust and the University of Birmingham, UK; Centre for Liver and Gastrointestinal Research, Institute of Immunology and Immunotherapy, University of Birmingham, UK; Liver Unit, University Hospitals Birmingham NHS Foundation Trust, Birmingham, UK.

Quentin M Anstee, Institute of Cellular Medicine, Faculty of Medical Sciences, Newcastle University, Newcastle upon Tyne, United Kingdom; Newcastle NIHR Biomedical Research Centre, Newcastle upon Tyne Hospitals NHS Foundation Trust, Newcastle upon Tyne, United Kingdom.

Rajiv Jalan, Liver Failure Group, Institute for Liver and Digestive Health, University College London, Royal Free Campus, London, United Kingdom

Ramon Bataller, Division of Gastroenterology, Hepatology and Nutrition, University of Pittsburgh Medical Center, Pittsburgh, PA, USA.

Rohit Loomba, Division of Epidemiology, Department of Family Medicine and Public Health, University of California at San Diego, La Jolla, California.

Silvia Sookoian, Department of Clinical and Molecular Hepatology, National Scientific and Technical Research Council (CONICET), University of Buenos Aires, Institute of Medical Research (IDIM), Ciudad Autónoma de Buenos Aires, Argentina.

Shiv K Sarin, Department of Hepatology, Institute of Liver and Biliary Sciences, New Delhi, India.

Stephen Harrison, Radcliffe Department of Medicine, University of Oxford, Oxford, UK.

Takumi Kawaguchi, Division of Gastroenterology, Department of Medicine, Kurume University School of Medicine, Kurume, Japan.

Vincent Wai-Sun Wong, Department of Medicine and Therapeutics, The Chinese University of Hong Kong, Hong Kong

Vlad Ratziu, Sorbonne Université, Assistance Publique-Hôpitaux de Paris, Hôpital Pitié Salpêtrière -, Institute of Cardiometabolism and Nutrition (ICAN), Paris, France.

Yusuf Yilmaz, Department of Gastroenterology, School of Medicine, Marmara University, Istanbul, Turkey. Institute of Gastroenterology, Marmara University, Istanbul, Turkey.

Zobair Younossi, Center for Liver Diseases, Department of Medicine, Inova Fairfax Hospital, Falls Church, VA, USA. 
Key words: MAFLD, nomenclature, metabolic, heterogeneity

\section{Abstract}

Fatty liver associated with metabolic dysfunction is common, affects a quarter of the population, and has no approved drug therapy. While pharmacotherapies are in development, response rates appear modest. The heterogeneous pathogenesis of metabolic fatty liver diseases and inaccuracies in terminology and definitions necessitate a reappraisal of nomenclature to inform clinical trial design and drug development. A group of experts sought to integrate current understanding of patient heterogeneity captured under the acronym nonalcoholic fatty liver disease (NAFLD) and provide suggestions on terminology that more accurately reflects pathogenesis and can help in patient stratification for management. Experts reached consensus that NAFLD does not reflect current knowledge and metabolic (dysfunction) associated fatty liver disease "MAFLD" was suggested as a more appropriate overarching term. This opens the door for efforts from the research community to update the nomenclature and sub-phenotype the disease in order to accelerate the translational path to new treatments. 


\section{Introduction}

\section{Why it is time to revise the fatty liver nomenclature?}

Since the term non-alcoholic fatty liver disease (NAFLD) was coined by Ludwig and colleagues in 1980 to describe fatty liver disease arising in the absence of significant alcohol intake $^{1}$, the nomenclature and criteria for a diagnosis has not been revisited. Yet, this disease has risen in prevalence, with a major impact on clinical and economic burden to society, such that nearly 1 billion people globally are affected ${ }^{2}$. Of concern, NAFLD is increasingly recognised and diagnosed in children and adolescents ${ }^{3}$, and this, when paired with the intimately associated hepatic as well as cardiovascular and oncological sequlae $e^{4,5}$, places an enormous burden on individuals, families and health-care systems ${ }^{6}$. The estimated annual medical costs directly attributable to NAFLD exceeds $€ 35$ billion in four large European countries (The United Kingdom, France, Germany and Italy) and \$100 billion in the United States $^{7}$. While reducing disease burden through prevention seems obvious, this have not been achieved. Further, while pharmacotherapies are expected to become available in the near future, none to date has been approved. Thus far, several phase $2 \mathrm{~b}$ and phase 3 studies either have fallen short of meeting current required histologic endpoints, or have done so with a modest margin. Muted efficacy of various compounds in development are in part a reflection of the imprecise definitions and the lack of precision medicine including consideration of heterogeneity of the disease.

Despite these alarming data, the nomenclature of the disease and the criteria for diagnosis have not been updated to reflect our expanding knowledge. The heterogeneity of the population with

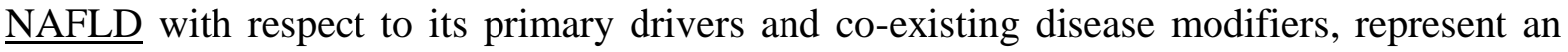
important impediment to the discovery of highly effective drug treatments. The phenotypic manifestation of fatty liver diseases likely reflects the sum of the dynamic and complex systems 
level interactions of these drivers; it follows that effective treatment requires that they be targeted with precision, based on a person's phenotype and genetic background ${ }^{8,9}$. However, trial recruitment is currently based on histologic grading and staging - and that is a problem because many pathways lead to the same histologic phenotype, without dissection of the predominant pathogenic pathways ${ }^{10,11}$. Perhaps not surprisingly, the response rates to current investigational agents range from 20 to $40 \%$ with a difference from placebo of $10-20 \%{ }^{8}$. Thus, a "one size fits all approach" would seem inappropriate when dealing with a very heterogeneous liver disease.

From the patient's perspective, the term 'non-alcoholic fatty liver disease' not only trivialises the problem by including terms such as 'non', but is also pejorative as it introduces words such as 'alcoholic' potentially placing the blame on the patient as having caused their condition. It also implies that the treatment must entirely lie in the patient's hand. This has enormous implications on how industry and policy makers choose to allocate resources for tackling the syndrome, which clearly is a major cause of death. Lessons can be learnt from cardiologists, diabetologists, neurologists and oncologists who have successfully distanced the disease they are trying to treat from the underlying obesity, smoking, alcohol abuse and drug abuse. Some of these factors have high genetic predisposition. In support of this idea, a meeting organised by the European Liver Patient's Association (ELPA) with the European Commission in 2018 suggested that a change in nomenclature was one of their key requirements.

As a first step to tackle this challenge, revising the nomenclature and definitions of the disease is critical. Recently, concerns over the inaccuracies of the nomenclature of fatty liver disease have been raised by individual experts ${ }^{12-14}$. In prior work, we called for a consensus to consider these aspects ${ }^{15}$ and in this review, an international panel sought to integrate epidemiological knowledge about disease progression that includes steatosis and steatohepatitis associated with metabolic dysfunction, with information about risk prediction derived from genetic and 
phenotyping studies. We suggest a new nomenclature based on consensus voting by participants to describe the disease that will allow us to properly sub-phenotype and stratify patients, via the application of more precise genetic, anthropometric and metabolic phenotyping approaches. In turn, detailed phenotyping will translate into individualised risk prediction and prevention strategies, and improvements in clinical trial design.

\section{Methods}

Following discussions, an initial concept sheet was circulated to the panel of contributors. This revealed widespread agreement and consensus that it was time to revisit the nomenclature of metabolic fatty liver disease as a critical initial step for improved patient sub-phenotyping, clinical trials design and ultimately, for personalisation of medicine.

Subsequently, a manuscript was drafted, circulated to the panel, and feedback incorporated over several rounds of revision. To reach consensus on a nomenclature, the Delphi method was adopted in two rounds. This method is a recommended iterative process for use in the healthcare setting as a reliable means to solicit and distil the judgments of experts and to

determine consensus via a systematic progression of repeated rounds of voting ${ }^{16}$. A "closed" electronic survey URL was sent to participants providing a unique link that could only be used once. Survey data were collected and managed using REDCap (Research Electronic Data Capture). In the first round of surveys members suggested one or more terms to describe metabolic fatty liver disease. In a second round (based on a summary of the experts' suggestions), participants were asked to vote on the suggested terminology. To ensure a robust and transparent process, anonymity of the participants was maintained.

\section{Metabolic associated fatty liver disease: a heterogeneous phenotype}

We now recognise that metabolic fatty liver disease is a phenotype with complex and disparate causes; the current terminology (NAFLD) represents an umbrella term for the multiple 
underlying sub-types ${ }^{17,18}$. This is evidenced by the wide spectrum of disease severity and natural history, as well as the substantial inter-patient variability across the spectrum. Although hepatic steatosis is highly prevalent, only a minority exhibit inflammatory injury at any time; more importantly, an individual can oscillate between steatosis and steatohepatitis even over a short timeframe ${ }^{19}$. In addition, while there is convincing evidence that liver-related complications (i.e., cirrhosis and cancer) are more likely in those with steatohepatitis, progression is far from inevitable ${ }^{19-21}$. Further, there is growing evidence that hepatocellular carcinoma (HCC) can develop in a fatty liver in the absence of cirrhosis ${ }^{22}$. Even among those with steatohepatitis, there appear to be individuals with apparent rapid-fibrosis progression and those with inherently slow-fibrosis progression ${ }^{23}$. Finally, disease evolution can be modified by exogenous interventions (for instance, life-style changes) ${ }^{24}$, superimposed disease states (e.g., type 2 diabetes mellitus) ${ }^{25}$, inherited predisposition ${ }^{26}$, and can even "spontaneously" regress, as has been demonstrated in placebo group participants in treatment trials and by observational dual-biopsy studies in secondary/tertiary care settings ${ }^{23,27,28}$. Adding to the complexity, it is unknown if the propensity for metabolic fatty liver diseases progression can vary across the lifespan. For example, given the rapidly escalating prevalence of metabolic fatty liver disease in children and young adolescents, we still do not understand if their natural history follows a different trajectory from those who develop disease in adulthood, middle age or even old age ${ }^{29}$.

\section{Sources of heterogeneity}

The heterogeneity in clinical presentation and disease course of fatty liver disease is likely influenced by multiple factors including age, gender, hormonal status, ethnicity, diet, alcohol intake, smoking, genetic predisposition, the microbiota and metabolic status. Thus, the final outcome will reflect the balance of these diverse inputs, each interacting with the other and modifying the ultimate manifestations and clinical course (Figure 1). It follows that effective 
treatment will require systematic dissection of the pathways involved and likely multifaceted and personalised treatments $\mathrm{s}^{30,31}$. A brief summary of current knowledge about factors contributing to NAFLD heterogeneity is provided below.

\section{Age and gender}

NAFLD prevalence, the risk of hepatic and extra-hepatic complications, and the likelihood of overall and disease-specific mortality increases with advancing age 19,21,32,33. With ageing, substantial changes occur in the liver including a decline in hepatic blood flow, hepatic volume, and liver function, a reduction in bile acid synthesis and alterations in cholesterol metabolism, as well as a reduction in mitochondrial number with subsequent increases in oxidative respiration $^{34}$. Cellular senescence has also been implicated ${ }^{35,36}$. Furthermore, ageing is accompanied by changes in body composition, including a decrease in muscle mass, an increase in abdominal adiposity and ectopic fat deposition, with increases in insulin resistance and prevalence of the metabolic syndrome ${ }^{37,38}$. Emerging evidence suggests that sarcopenia is associated with both NAFLD and NAFLD-related advanced fibrosis, even after adjusting for BMI and insulin-resistance ${ }^{39,40}$. Presumably, ageing also captures greater exposure to the drivers, which result in steatohepatitis and fibrosis.

Equally, as recently reviewed ${ }^{41}$, there is substantial sexual dimorphism in many aspects of fatty liver disease with regard to risk factors, prevalence, fibrosis pattern, and disease outcomes. Generally, prevalence tends to be lower in women predominantly at earlier disease stages, whereas, disease frequency increases in postmenopausal women ${ }^{41}$. Similarly, fatty liver prevalence is lower in post-menarchal girls than in boys ${ }^{42}$. Among postmenopausal women, those not on hormone replacement therapy (HRT) tend to have higher disease prevalence compared to those on $\mathrm{HRT}^{43}$, and similarly, premenopausal women have less severe hepatic

fibrosis and better survival compared to men and postmenopausal women ${ }^{44,45}$. Consistently, a 
longer duration of estrogen deficiency associates with a higher likelihood of fibrosis among postmenopausal women with fatty liver disease ${ }^{46}$. By analogy, studies of diet induced mouse models suggests that males develop more severe steatosis and liver histology compared to females 47,48 .

Although the mechanisms for these effects are not completely understood, sex differences in adiposity, metabolic risk factors and body fat distribution (which tends to shift towards abdominal obesity after menopause), likely play a role ${ }^{49}$. A recent study in mice from $\sim 100$ strains included in the hybrid mouse diversity panel (HMDP) demonstrated that multiple molecular pathways and gene networks implicated in lipid metabolism, insulin-signalling and inflammation show sexual dimorphism ${ }^{50}$. Similarly, another study demonstrated sexual differences in liver gene expression of regulators of multiple metabolic pathways using a mice computational model. Notably, some such as peroxisome proliferator-activated receptor PPAR $\alpha$, farnesoid $X$ receptor $(F X R)$ and liver $X$ receptor $(\mathrm{LXR})$, which are highly gender dependent, are currently being investigated as therapeutic targets for steatohepatitis ${ }^{51}$. A further study demonstrated gender-related pathways contribute to steatosis and fibrosis in male and female mice (males mainly inflammation and females mainly alterations of redox state), despite similar endpoints ${ }^{52}$. Clearly, sex and menopausal status influence disease outcomes and require stratification as treatment responses can vary substantially.

\section{Ethnicity}

Population-based data show ethnic differences in the prevalence of fatty liver; a recent metaanalysis demonstrated both NAFLD prevalence and risk of NASH were highest in Hispanics, intermediate in Whites, and lowest in Blacks. However, fibrosis risk did not differ according to ethnicity ${ }^{53}$. Metabolic fatty liver disease is also rapidly increasing in Asian populations ${ }^{54}$. Previous studies have demonstrated that Asians tend to accumulate liver fat at lower body mass 
index (BMI) compared to those of other races ${ }^{55}$. The course of disease also appears to be more severe in Asians compared to non-Asians, and they tend to have more lobular inflammation and higher grades of ballooning compared to other ethnicities ${ }^{56,57}$. While data regarding fibrosis are scarce in the studies above, Asians tended to have a higher risk of fibrosis, while Africans were at lower risk compared to whites; this did not reach significance, perhaps due to sample size limitations ${ }^{56,57}$. However, and notably, these biopsy-based studies might suffer from selection bias. For example, a community-based study in Hong Kong suggested that while NAFLD is detected in a quarter of the population, the prevalence of advanced fibrosis is $1 \mathrm{w}^{58}$. The reasons for racial disparities in fatty liver risk are not completely understood. Plausible explanations include variations in genetic predisposition, metabolic attributes, cultural and socioeconomic factors, dietary and exercise habits, access to health care as well as environmental risks. There are substantial differences in genetic heritage across ethnic groups; variation in the risk allele of the Patatin-like phospholipase domain-containing protein 3 (PNPLA3) gene that is most frequent in Hispanics (49\%), followed by non-Hispanic white (23\%) and African Americans (17\%) has helped, at least partially, to explain some of this ethnic variability ${ }^{59-61}$. In addition, the risk allele of the PNPLA3 rs738409 polymorphism was found to be more common in East Asians than Caucasians ${ }^{62}$. Notably, because the effect size of fatty liver-related gene variants supports the existence of differences among races, the relative contribution of specific genetic and and environmental triggers (e.g. dietary factors) or modifying risk variants, toward disease pathogenesis is likely variable among ethnic groups (Figure 2).

On the other hand, there are marked racial/ethnic socioeconomic disparities that are likely also reflected in differences in multiple disease risk factors. For example, there is a clear difference between European and Asian populations with regard to insulin resistance and body fat distribution, as discussed later. There are also disparities in physical activity; in 2016, a report 
including 1.9 million participants across 168 countries suggested that women in Latin America, south Asia, and high-income Western countries have the highest prevalence of physical inactivity ${ }^{63}$. Likewise, data from the NASH Clinical Research Network (NASH CRN) reported less physical activity, increased carbohydrate consumption and lower income levels in Hispanics compared with non-Hispanic white patients with $\mathrm{NASH}^{64}$. A role for gut microbiota could also be implicated, as discussed below.

\section{Light and moderate alcohol use}

Since its first description, metabolic has been considered distinct from alcoholic associated liver disease based on a cut-off of daily alcohol intake of $30 \mathrm{~g}$ daily for men and $20 \mathrm{~g}$ daily for women. The assumption underlying the cut off has been that alcohol intake below these thresholds does not induce hepatic steatosis or have deleterious impacts on liver disease progression and outcomes ${ }^{65}$.

Due to the high prevalence of adults with NAFLD who drink at least in moderation $(\sim 4$ drinks/week $)^{66}$, there is now much interest in the influence of light and moderate alcohol use on the prognosis of NAFLD, with debate on the protective effects ${ }^{67,68}$ and perceived harms ${ }^{69}$,

70. More recently, there has been evidence for and against safe limits for alcohol consumption in the setting of NAFLD ${ }^{71}$. Some reports suggest that modest alcohol consumption, even after adjustment for previous heavy drinking, is associated with a reduction in vascular complications $^{67,72}$ or has no impact ${ }^{73}$. Other studies have demonstrated that moderate drinking ( 2 drinks a day for women and 3 drinks a day for men) is associated with a reduced prevalence of NASH and advanced fibrosis ${ }^{74}$. In contrast some studies highlight that even low alcohol intake in those with a fatty liver is associated not only with increased risk of disease progression, but also for advanced liver disease and cancer ${ }^{75,76,77,78}$ and decreased rates of improvement in steatosis and NASH ${ }^{79}$. The effect of alcohol use on liver disease evolution 
likely has a dose-response, rather than a J-shaped association ${ }^{80,81}$, with a synergistic detrimental effect with the presence of metabolic syndrome ${ }^{75,77}$ as has recently been reviewed 74.

\section{Dietary intake, gut microbiota and bile acids}

For metabolic homeostasis, the neuroendocrine axis, dietary intake, muscle mass, physical activity, and the enterohepatic circulation, gut microbiota, bile acids and their related metabolites are intimately implicated in fatty liver pathogenesis (Supplementary Figure 1). The dietary pattern that characterizes the Western diet, including increased fat and fructose consumption that is fuelling the increase in obesity and fatty liver, is associated with a wide range of metabolic dysfunction, including insulin resistance and abnormal lipid profile ${ }^{82}$. In contrast, adoption of a Mediterranean dietary pattern is accompanied with a decrease in liver fat in patients with NAFLD and a decrease in cardiovascular risk ${ }^{83,84}$.

Microbiota composition can change rapidly and widely according to dietary patterns ${ }^{83,85}$ and the involvement of the gut microbiome in fatty liver and steatohepatitis in both mice and humans is well recognised ${ }^{86,87}$. Emerging data suggest that the microbiome and gut microbiome-derived metabolites can predict advanced fibrosis and cirrhosis in NAFLD ${ }^{88-91}$. Gut microbiota are also implicated in regulating bile acids and their metabolites, which in turn regulates glucose, lipid and choline metabolism, and energy homeostasis ${ }^{92}$. Altered gut flora and intestinal permeability have also been shown in patients and murine models of NAFLD ${ }^{93}$, ${ }^{94}$. This leads to increased circulating levels of bacterial products including lipopolysaccharide (LPS) as well as other bioactive compounds that may induce intra-hepatic activation of proinflammatory cells, hepatic stellate cells and hepatocytes via stimulation of toll-like receptors (TLRs; particularly 2, 4 and 9), a sensor for these products ${ }^{95-97}$. However, it remains challenging to disentangle the effects of diet and its associated consequences for liver disease, from effects mediated by diet-induced alterations to the microbiome, and to ascertain causality 
under these same conditions. Notably, a role for human genetic variation and ethnicity in driving differences in microbiomes has recently been suggested ${ }^{98-100} 101$.

\section{Obesity and metabolic health}

Although obesity intimately associates with liver fat, not all patients with obesity develop metabolic fatty liver disease ${ }^{2}$. Whereas obesity can be classified as metabolically healthy obesity (MHO) and metabolically unhealthy obesity (MUO), with the former affecting about $45 \%$ of obese subjects, there is no consensus on a definition of metabolic health. Various definitions of metabolic syndrome include a combination of different metabolic components 102, 103. Similarly, while insulin resistance is believed to play a pivotal role and is a pathophysiological feature of fatty liver ${ }^{104}$ it has not been included in several definitions of metabolic syndrome. Notably, multiple large-scale cohort studies do not clearly support the notion that metabolically healthy obesity subgroups, at least as currently defined, are protected from cardiometabolic complications compared with those with a stable normal weight who are metabolically healthy ${ }^{105-107}$. Better classification based on molecular or genetic profiling could help dissect with high precision, metabolically favourable and unfavourable subtypes, with distinct metabolism, anthropometry and patterns of fat deposition, and likely differential responses to drug treatments ${ }^{108}$. On the other hand, $\sim 30 \%$ of normal-weight individuals can be classified as metabolically obese normal weight (MONW) and they demonstrate an increased propensity for cardiometabolic risk; a fair proportion of patients with a fatty liver are also lean.

Current consensus suggests that the distribution and the overall health of fat, rather than its amount is likely the major determinant of disease risk. For example, higher amounts of visceral relative to peripheral and subcutaneous adipose tissue is associated with greater metabolic risk 109,110 and is directly linked to liver inflammation and fibrosis, independent of insulin resistance and hepatic steatosis ${ }^{109}$. Sex, sex hormones (as discussed above), ethnicity and genes obviously 
play important roles in determining the location and health of adipose tissue. There is for example, strong evidence that ethnicity is implicated in determining fat distribution and health 111. Thus, abdominal and visceral adiposity are greater among Asians compared with Caucasians, and lower in Africans ${ }^{112-115}$ as is insulin resistance despite an equal or lower BMI 116117,118 . Genetic variants also play a role in the regulation of fat distribution ${ }^{119,120,121}$, with “favourable adiposity" genes have been recently identified ${ }^{103,122,123 .}$

Although lipid accumulation in liver is a hallmark of NAFLD, there is emerging evidence that there is likely a variety of underlying mechanisms and routes for its development. For instance, a recent study has demonstrated that lipid composition in liver is very different in two proposed sub-types of NAFLD. In sub-type 1 based on insulin resistance, patients tend to have monounsaturated TAGs and free fatty acids enriched with ceramides in liver, while sub-type 2 based on carrying the PNPLA3 risk genotype at rs738409, have polyunsaturated triacylglycerols (TAG) ${ }^{124}$. Similarly, another study suggested the existence of three NAFLD subtypes, with different metabolic phenotypes ${ }^{125}$. In another study, regions with steatosis demonstrated distinct lipid composition, predominantly in the form of a loss of arachidonic acid-containing intracellular phospholipids, compared to non-steatosis liver tissue ${ }^{126}$. A further report used RNAseq analysis identified molecular subtypes with distinct gene expression pattern clusters that are implicated in lipid metabolism, interferon signalling and immune system pathways, according to different histological scores ${ }^{127}$. In total, these new datasets emphasize that there are likely multiple NAFLD subtypes characterized by unique metabolomic signatures. Based on subtype, it is likely that treatment responses will vary and hence defining the metabolic landscape of an individual is likely important in clinical trial design. 


\section{Lean NAFLD}

Currently, lean NAFLD, or NAFLD in lean individuals, is defined as hepatic steatosis with a BMI $<25 \mathrm{~kg} / \mathrm{m}^{2}$ (or $<23 \mathrm{~kg} / \mathrm{m}^{2}$ in Asians) in the absence of 'significant' alcohol intake. ${ }^{128}$ Though first described in Asian populations, it is recognised that between 5\% and $45 \%$ of patients with NAFLD are lean; even among Europeans, about $20 \%$ of patients are considered lean ${ }^{129}$. Although those with lean NAFLD have a better metabolic and histological profile compared to their counterpart obese subjects, their natural history is poorly defined, with some data suggesting they may have a worse outcome and accelerated disease progression ${ }^{130,131}$, while others suggest no difference or even better outcomes ${ }^{132,133}$. More recent data proposes that lean NAFLD comprises a distinct pathophysiological entity from that in obese subjects, which extends beyond just simple differences in BMI. In that study, lean patients had distinct metabolic and gut microbiota profiles compared to their obese counterparts and lean healthy controls. Specifically, they had intact metabolic adaptation in response to an obesogenic environment via increased bile acids and FXR activity that likely helped them to maintain an obesity-resistant phenotype. Notably, either this adaptation tends to be lost with advancement of disease or the failure to adapt promotes disease progression. Other intriguing aspects from a subset of the patients suggests that they have a distinct gut microbiota profile, with enrichment of species implicated in the generation of liver fat, and a genetic profile with an increased prevalence of the TM6SF2 risk allele ${ }^{134}$, as also observed by another study ${ }^{135}$. Further studies will be required to explore whether the metabolic adaptation observed in lean NAFLD is seen in other subtypes of patients.

\section{Familial Risk}

Data from well-characterized cohorts of twins who underwent imaging to quantify liver fat and fibrosis has shown that both are heritable traits ${ }^{136}$. Furthermore, retrospective family-based studies show that there is familial aggregation of NAFLD and cirrhosis ${ }^{137}$. Consistently, a 
recent prospective study including probands with NAFLD-cirrhosis and their first-degree relatives indicated that the risk of advanced fibrosis among first-degree relatives of patients with cirrhosis is $18 \%^{138}$. This is substantially higher than the risk of cirrhosis in the general population and points towards further sub-stratification of the population by family history of cirrhosis due to NAFLD.

\section{Genetic variation}

Genome-wide association and large candidate studies have identified multiple loci associated with NAFLD and NASH. While in depth discussion is beyond our scope, the topic has recently been reviewed ${ }^{139,140}$. At least five common variants in different genes have been associated with NAFLD, namely PNPLA3, transmembrane 6 superfamily member 2 (TM6SF2), glucokinase regulator $(G C K R), M B O A T 7$, and hydroxysteroid 17-beta dehydrogenase-13 $(H S D 17 B 13)^{140}$. Multiple other genes have reported associations, including polymorphisms in inflammatory, immune and metabolism-related, oxidative stress, adipokine, and myokinerelated genes ${ }^{139-144}$. It is noteworthy however that all known variants explain only a small proportion of NAFLD, suggesting the existence of heritability factors that are yet to be defined 145. Exploring the role of other types of genetic variation, gene-gene and gene-environment interactions, epigenetics, common variants that do not reach genome-wide significance, and rare and less common variants will help dissect the missing heritability ${ }^{146,140,147}$. For example, a gene-environment interaction has been proposed for the PNPLA3 variant with dietary patterns ${ }^{148}$, increased intake of sugars ${ }^{149}$, omega- 6 poly-unsaturated fatty acids intake ${ }^{150}$, obesity, and insulin resistance ${ }^{151}$.

Of interest, described NAFLD-related variants show divergent metabolic effects. Multiple reports indicate an association of a genetic variant of TM6SF2 (encoding p.Glu167Lys) with lower serum lipid levels and lower risk of coronary artery disease, but with increased risk of 
fatty liver and advanced fibrosis ${ }^{152-154}$, even in those with viral hepatitis ${ }^{155}$. Although early reports suggested that PNPLA3 rs738409 has no association with the metabolic profile ${ }^{156}$, more recent larger studies and a Phenome-wide association study (PheWAS) study indicate that it has similar metabolic effects to TM6SF2 rs58542926 ${ }^{157,158}$. An association of PNPLA3 rs738409 and TM6SF2 rs58542926 with type 2 diabetes has also been demonstrated beside the known association of GCKR rs1260326 with diabetes ${ }^{159}$. Variants in HSD17B13 and MBOAT7 do not to date appear to have an effect on serum lipids, glycaemia or risk of coronary heart disease ${ }^{160-163}$.

\section{Epigenetic factors}

Reversible epigenetic changes represent a plausible bridge between genes and the environment; their dysregulation is implicated in several diseases, including NAFLD ${ }^{140}$. Numerous microRNAs (miRNAs) have been linked to NAFLD. A recent meta-analysis demonstrated that in particular, miRNA-122, miRNA-34a and miRNA-192 could be biomarkers of fatty liver disease ${ }^{164,165}$. miRNA-122 and miRNA-192 showed upregulation in NAFLD compared to healthy controls while miRNA-34a was upregulated in NAFLD and correlated with disease severity ${ }^{164,165}$.

Data on the role of long non-coding RNAs (lncRNAs) and other type of non-coding RNAs in NAFLD is limited. Some data suggests alterations in lncRNAs in NASH, such as a hepaticspecific lnc18q22.2 ${ }^{166}$, a brown fat-enriched lncRNA 1 (Blnc1), ${ }^{167}$ and metastasis-associated lung adenocarcinoma transcript 1 (MALAT1) ${ }^{168}$. A study using genome scanning with next generation sequencing has identified other candidates ${ }^{169}$. The role of lncRNAs in steatohepatitis remains to be further elucidated in larger cohorts.

Several studies show wide alterations in the methylation signature of hepatic as well as peripheral blood-derived DNA, including regulatory loci for key metabolic, inflammatory, and 
fibrotic pathways, in patients with NAFLD. Some of these signatures appear to reverse following bariatric surgery ${ }^{170,171,172}$. There is also evidence that DNA methylation can be a biomarker for fibrosis stratification in NAFLD ${ }^{173}$ and that it regulates the expression of PNPLA3 ${ }^{174}$. For example, hypermethylation of the PPAR $\gamma$ promoter can be used to identify patients with advanced fibrosis ${ }^{173}$. More recently, a series of studies have shown evidence of methylation of the key mitochondrial urea cycle enzymes carbamoyl phosphate synthase- 1 and ornithine transcarbamylase enzymes resulting in a reduction in their function and hyperammonemia in NAFLD patients ${ }^{175}$. Hyperammonemia activates stellate cells and is associated with progression of fibrosis in NAFLD ${ }^{176,177}$; treatment of hyperammonemia using ornithine phenylacetate prevented progression of fibrosis in an animal model, suggesting a potential novel metabolic therapeutic strategy ${ }^{178}$.

Importantly, epigenetic mechanisms play a crucial role in foetal metabolic programming of liver fat ${ }^{179,180}$, with growing evidence that the earliest origins of NAFLD extend to in utero experiences. Data from animals suggest that a maternal diet high in fat triggers widespread epigenetic alterations in foetal hepatic DNA, accompanied by metabolic maladaptation that favours an increase in the risk of developing NAFLD in the offspring ${ }^{181,182}$. Even paternal diet patterns and prediabetes increase the risk of diabetes in offspring ${ }^{183}$. Notably, these changes can be transmitted over generations, but can also be altered by exercise and lifestyle interventions ${ }^{184-186}$. Although data in humans are still limited, maternal obesity and patterns of infant nutrition are risk factors for the development of NAFLD in adolescence and adulthood. For instance, normal pre-gestational BMI and breast-feeding for more than 6 months reduces the risk of developing NAFLD in the mother during mid-life ${ }^{187}$ and during adolescence in offspring ${ }^{188}$. Similarly, an increase in methylation of the peroxisome proliferator-activated receptor $\gamma$ coactivator 1 (PGC1) gene that controls several aspects of 
energy metabolism in liver ${ }^{189}$ and in newborns, is correlated with increased maternal pregestational BMI ${ }^{190}$.

\section{Why do we need to consider NAFLD heterogeneity in clinical practice?}

\section{Impact on the performance of non-invasive assessment of fibrosis}

Non-invasive fibrosis scores are commonly used to identify or exclude significant or advanced fibrosis in patients with fatty liver disease. However, a recent study suggested that the performance of scores such as the NAFLD fibrosis score (NFS) and fibrosis 4 (FIB-4) may vary across the life span, with lower specificity among older adults and lower accuracy in young adults ${ }^{191}$. The performance of non-invasive scores and the used Transient Elastography liver stiffness cut offs in different ethnic populations and in special subpopulations such as diabetic and obese individuals also need to be considered. For example, it has been shown that blood biomarkers are less accurate in South Asians compared to Europeans, regardless of metabolic indices ${ }^{192}$. As it is likely that blood-based biomarkers or imaging techniques will supplant liver biopsy for the diagnosis of disease in patients who would benefit from drug treatment, equally it implies that any future marker should be validated in more precisely defined cohorts. Thus, the consensus group suggests that the factors that shape the heterogeneity of NAFLD be considered when devising and applying risk-stratification scores and algorithms. This approach will continue to evolve as new contributors to disease variability are identified.

\section{Impact on the development of clinically-relevant animal models}

The complexity of human NASH is paralleled by the heterogeneity of animal models and the inability of these models to replicate the gamut of disease ${ }^{193}$. This represents both a barrier to the development of novel therapeutics but also an opportunity to better understand steatohepatitis pathogenesis based on different drivers of disease. Considering that NAFLD as 
described today is not a single entity, exploring the overlapping features of preclinical models with subtypes of NAFLD may help in overcoming these challenges. For instance, it has been reported that the Methionine adenosyltransferase 1A (Mat1a) deficient mouse can recapitulate a subtype of human NAFLD ${ }^{125}$, while mice fed a high cholesterol or methionine/choline deficient diet seem to recapitulate several features of lean NAFLD ${ }^{194}$. Despite the range of available models, there remains a need to develop improved in vitro and in vivo model systems.

\section{Impact on clinical trials design and the ability to find treatments}

The growing magnitude of NAFLD and the lack of effective drug treatments is reflected in intense clinical trial activity that has jumped from just eight in 2013 to over 300 ongoing in $2018^{195}$. Unfortunately, response rates remains modest, with $<20-30 \%$ of participants demonstrating NASH resolution and fibrosis regression. This low response can be attributed to many factors, including heterogeneity in population selection, lack of stratification based on the underlying dominant driver mechanisms, and the Hawthorne (placebo) effect ${ }^{8}$. Therefore, the standard clinical trial design that does not take into consideration disease heterogeneity may not be the best option for studying a complex disease. Thus, future clinical trials will likely target patients with specific characteristics (sex, hormonal status, genetic predisposition, metabolic and microbiota signatures and the presence or absence of comorbid conditions) once the relationships between the characteristics and the treatment targets are understood. Such trial design will likely include rational combination approaches ${ }^{31}$.

Considering alternative innovative trial designs might be a viable option (Figure 3). Recently, using overarching or master protocols designed to address multiple questions by investigating different drugs (more than one or two therapies that might even include direct comparisons of competing drugs) in different conditions (more than one patient type or disease), all within the

same overall trial structure has been suggested ${ }^{196}$. Adaptive trial designs that provide flexibility 
for altering one or more aspects of the basic features of the study design based on responses in earlier phases is also an option ${ }^{197}$, although this will add substantial complexity to data interpretation. Notably, given the heterogeneity of NAFLD according to ethnicity and geographic region, regional stratification or performing separate trials in different geographic regions should be considered for key trials.

\section{Is NAFLD the right name for metabolic liver disease?}

How do the above considerations influence our thinking on the need to revise the definition and nomenclature for NAFLD? It is clearly the time to do this. The suggestion of this consensus focusses on four aspects.

First, NAFLD was described as a condition of "exclusion", which means that it exists only when other conditions such as viral hepatitis B and C, autoimmune diseases or alcohol intake above a particular threshold are absent. However, with advancements in our understanding of the underlying pathological processes, it is clearly a disease that must be defined by inclusion, rather than by exclusion. Further, given its high prevalence in most affluent populations, especially those consuming a westernized diet, fatty liver disease is recognized to coexist with other conditions such as viral hepatitis, autoimmune diseases and alcohol ${ }^{198-200}$ and will have synergistic effects on disease progression ${ }^{201,202}$. The nomenclature for fatty liver disease and criteria for diagnosis need to reflect this new knowledge.

Second, there remains debate about the safe limit of alcohol intake. Updating a diagnosis of NAFLD to zero or near to zero alcohol consumption as has been suggested by some is clearly impractical, as recently discussed ${ }^{15}$. Furthermore, there are significant methodological challenges in questionnaires used for measuring alcohol consumption including documenting prior and over life use, low amounts of intake, patient underreporting and recall bias, as well as marked variability in defining terms such as "social drinking" and "binging" in individuals 
with NAFLD. Thus, linking metabolic fatty liver disease, a distinct entity, to alcohol in its name is problematic. Moreover, including the term "non-alcoholic" in the name is disappointing for abstemious patients and links this entity to the stigma of alcohol consumption. Confounding terms in the name of these diseases should be replaced as has already been done with primary biliary cirrhosis becoming primary biliary cholangitis, with sometimes redundant but more accurate and clear words, defining the entity ${ }^{203}$. More importantly, there is an urgent need to identify coexisting metabolic and alcohol liver disease so that they may be treated appropriately. This group of patients is distinct from those with pure or predominant alcoholic cirrhosis. Such patients are currently excluded from all NASH trials.

Third, though in clinical practice we segregate patients into those with NASH and those without, whether this is appropriate is a matter of debate. As we know, there is tremendous plasticity in metabolic liver disease over the life span and strong evidence that fibrosis is the major determinant of adverse outcomes ${ }^{21}$. Hence, the current classification may be misleading and perhaps metabolic dysfunction associated fatty liver disease should be considered similar to other chronic liver diseases with some degree of activity and a stage of fibrosis, without dichotomous stratification into NASH and non-NASH. From a pathological perspective, this will result in improved disease classification, at least in the context of liver biopsy ${ }^{26}$.

Fourth, the heterogeneous nature of fatty liver diseases suggests that they cannot be considered or managed as a single condition with a "one size fits all" approach to therapy. Lack of consideration of heterogeneity impacts and detracts from our ability to precisely define the natural history of fatty liver phenotypes, to appropriately select for clinical trials that are weighted to demonstrate meaningful benefits, and to compare or pool results from the trials. For these reasons, an updated and appropriate nomenclature for the disease is the first step in the long path to deconvolution of disease heterogeneity. 
Based on the above, participants agreed on the need for a revised and updated terminology; the bulk of respondents in the first round of survey suggested that the words metabolism, fat and liver be included in some form in the name. The final vote favouring Metabolic Associated Fatty Liver \pm Disease (MAFL/MAFLD) (supported by $72.4 \%$ of participants). The second preference Metabolic Fatty Liver +/- Disease (MEFL/MEFLD) was supported by $17.2 \%$ (Supplementary table 1). Thus, the panel suggests we eliminate the term "NAFLD" from the lexicon and replace it with metabolic associated fatty liver "MAFLD". The term MAFLD represents the overarching umbrella of the common disease we treat and will have multiple sub-phenotypes, reflecting the dominant driver of disease. Obviously, many, if not most, patients will have overlapping contributions from other and distinct liver diseases that range from alcohol (regardless the amount) to viral hepatitis. The natural history of these latter groups is likely very different from those with pure metabolic dysfunction.

\section{Conclusion}

The outdated NAFLD/NASH acronyms, the criteria for diagnosis and a lack of adequate consideration of heterogeneity in risk profiles and treatment responsiveness represent barriers that hamper progress towards effective treatments. The consensus group has suggested an acronym (MAFLD) that we believe more accurately reflects current knowledge of fatty liver diseases associated with metabolic dysfunction that should replace NAFLD/NASH. In addition, we have identified gaps in current knowledge and highlight new strategies and tools to overcome the challenges (Supplementary table 2). A summary of suggestions is provided in Table 1. The group acknowledges the many investigators in the field who have made similar well-reasoned pleas for a change in nomenclature. This work also opens up for wider consultation with the public, patients, regulators and non-hepatology health care workers, the necessity for a nomenclature update. Future studies will allow us to further characterise and 
sub-phenotype the disease and its drivers as a necessary prerequisite for the design of more appropriate clinical trials and for patient management and to consider the implications of the updated of nomenclature on clinical practice and public health policy (Figure 4). 


\section{Figures legends}

Figure 1: Heterogeneity of metabolic associated fatty liver disease. The heterogeneity in clinical presentation and course of fatty liver disease is influenced by a multitude of factors including age, sex, ethnicity, alcohol intake, dietary habits, hormonal status, genetic predisposition and epigenetic factors, the microbiota and metabolic status. It is likely that there is a differential impact in the contribution of the various factors in any individual over time and among individuals that then shapes disease phenotype and course.

\section{Figure 2: Inter-individual variation in the predominant drivers of metabolic associated} fatty liver disease. Metabolic associated fatty liver disease is a complex phenotype shaped by the dynamic interaction of genetic predisposition with environmental factors and components of the metabolic syndrome. The effect size of genetic variants and the predominant drivers can exhibit marked inter-individual variation. As an example, disease in patient 1 is driven predominantly by environmental influences with less contribution from genetic predisposition; in patient 2, metabolic syndrome is the predominant driver, while disease in patient 3 is driven by genetic factors with a limited contribution from other factors. Identification of the predominant drivers in every patient can help in personalisation of medicine.

Figure 3: Innovative clinical trials for metabolic associated fatty liver disease. The substantial heterogeneity of patients with metabolic associated fatty liver disease and the limited responses to investigational targets in current clinical trials imply that innovative trial designs are required. Trial designs such as umbrella, basket and adaptive designs have been suggested to overcome the challenges. However, such designs add complexity to the trial analysis.

Figure 4: Implications of the proposed update to the metabolic associated fatty liver disease nomenclature. The growing burden of metabolic associated fatty liver disease in the 
absence of effective therapies requires an updated process map to address the challenges. The first step is an update of nomenclature, as without precise terminology, neither patient care nor science can be adequately served. This update of nomenclature we expect will be a step towards further characterisation of disease heterogeneity. In turn, detailed phenotyping can guide the development of better preclinical models and identify novel therapies that are likely to be effective for particular patient subtypes, but not others. This will lead to improved clinical trial designs, allowing us to compare and pool results and thereby help reduce the impact of disease burden.

\section{Supplementary Figure 1: Conceptual framework of metabolic dysfunction and} pathogenesis of metabolic associated fatty liver disease. For metabolic homeostasis, the neuroendocrine axes elicits multiple and complex responses that orchestrate with caloric intake, muscle mass and physical activity as well as with the enterohepatic circulation, including gut microbiota, bile acids and their metabolites. These circles are interconnected at various levels. For example adiponectin signaling from adipose tissue to liver, the liver (FGF 21) to the central nervous system, the duodenum (Cholecystokinin) to the brain, etc. These various inputs are integrated in the liver. Dysfunctional homeostatic responses at any of multiple levels are implicated in the heterogeneous pathogenesis of metabolic associated fatty liver disease. 
Table 1: Statements of the consensus panel

Nomenclature and definition of metabolic associated fatty liver disease (MAFLD)

- We suggest that the nomenclature of NAFLD should be updated to MAFLD.

- The diagnosis of MAFLD should be based on the presence of metabolic dysfunction not the absence of other conditions

- MAFLD can co-exist with other liver diseases

- A reference to alcohol should not be included in the MAFLD acronym.

- Patients with both MAFLD and a contribution from alcohol to their liver disease represent a large and important group that requires further investigation and characterisation.

\section{MAFLD heterogeneity}

- MAFLD is a heterogeneous entity

- Appropriate patient stratification must be considered when non-invasive fibrosis scores are developed and in clinical trial design

- Studies are required to map the landscape of MAFLD and to precisely define subtypes of the disease

\section{Clinical trials for MAFLD}

- Detailed patient stratification and tailoring clinical trial inclusion criteria based on drivers of disease will likely yield more informative and meaningful results

- Innovative designs for clinical trials and personalised combination therapy approaches will likely be required to overcome the challenges of disease heterogeneity and for optimal clinical efficacy. 


\section{References}

1. Ludwig J, Viggiano TR, McGill DB, et al. Nonalcoholic steatohepatitis: Mayo Clinic experiences with a hitherto unnamed disease. Mayo Clin Proc 1980;55:434-8.

2. Younossi Z, Anstee QM, Marietti M, et al. Global burden of NAFLD and NASH: trends, predictions, risk factors and prevention. Nature Reviews Gastroenterology \& Hepatology 2018;15:11-20.

3. Wiegand S, Keller KM, Robl M, et al. Obese boys at increased risk for nonalcoholic liver disease: evaluation of 16390 overweight or obese children and adolescents. International Journal of Obesity 2010;34:1468-1474.

4. Jepsen $\mathrm{P}$, Vilstrup $\mathrm{H}$, Mellemkjaer $\mathrm{L}$, et al. Prognosis of patients with a diagnosis of fatty liver A registry-based cohort study. Hepato-Gastroenterology 2003;50:2101-2104.

5. Sorensen HT, Mellemkjaer L, Jepsen P, et al. Risk of cancer in patients hospitalized with fatty liver - A Danish cohort study. Journal of Clinical Gastroenterology 2003;36:356-359.

6. Younossi Z, Tacke F, Arrese M, et al. Global Perspectives on Nonalcoholic Fatty Liver Disease and Nonalcoholic Steatohepatitis. Hepatology 2019;69:2672-2682.

7. Younossi ZM, Blissett D, Blissett R, et al. The economic and clinical burden of nonalcoholic fatty liver disease in the United States and Europe. Hepatology 2016;64:1577-1586.

8. Friedman SL, Neuschwander-Tetri BA, Rinella M, et al. Mechanisms of NAFLD development and therapeutic strategies. Nature Medicine 2018;24:908-922.

9. Eslam M, George J. Genetic Insights for Drug Development in NAFLD. Trends Pharmacol Sci 2019;40:506-516.

10. Buzzetti E, Pinzani M, Tsochatzis EA. The multiple-hit pathogenesis of non-alcoholic fatty liver disease (NAFLD). Metabolism-Clinical and Experimental 2016;65:1038-1048.

11. Skoien R, Richardson MM, Jonsson JR, et al. Heterogeneity of fibrosis patterns in non-alcoholic fatty liver disease supports the presence of multiple fibrogenic pathways. Liver International 2013;33:624-632.

12. Dufour JF. Time to Abandon NASH? Hepatology 2016;63:9-10.

13. Bellentani S, Tiribelli $C$. Is it time to change NAFLD and NASH nomenclature? Lancet Gastroenterology \& Hepatology 2017;2:547-548.

14. Loria P, Lonardo A, Carulli N. Should nonalcoholic fatty liver disease be renamed? Digestive Diseases 2005;23:72-82.

15. Eslam M, Sanyal AJ, George J. Toward More Accurate Nomenclature for Fatty Liver Diseases. Gastroenterology 2019;157:590-593.

16. Dalkey NC. STUDIES IN THE QUALITY OF LIFE; DELPHI AND DECISION-MAKING, 1972.

17. Yki-Jarvinen $\mathrm{H}$, Luukkonen PK. Heterogeneity of non-alcoholic fatty liver disease. Liver Int 2015;35:2498-500.

18. Lonardo A, Ballestri S, Targher G. "Not all forms of NAFLD were created equal". Do metabolic syndrome-related NAFLD and PNPLA3-related NAFLD exert a variable impact on the risk of early carotid atherosclerosis? Atherosclerosis 2017;257:253-255.

19. Adams LA, Lymp JF, St Sauver J, et al. The natural history of nonalcoholic fatty liver disease: a population-based cohort study. Gastroenterology 2005;129:113-21.

20. Angulo P, Kleiner DE, Dam-Larsen S, et al. Liver Fibrosis, but No Other Histologic Features, Is Associated With Long-term Outcomes of Patients With Nonalcoholic Fatty Liver Disease. Gastroenterology 2015;149:389-397 e10.

21. Vilar-Gomez E, Calzadilla-Bertot L, Wong VWS, et al. Fibrosis Severity as a Determinant of Cause-Specific Mortality in Patients With Advanced Nonalcoholic Fatty Liver Disease: A MultiNational Cohort Study. Gastroenterology 2018;155:443-+. 
22. Mittal S, El-Serag HB, Sada YH, et al. Hepatocellular Carcinoma in the Absence of Cirrhosis in United States Veterans Is Associated With Nonalcoholic Fatty Liver Disease. Clinical Gastroenterology and Hepatology 2016;14:124-+.

23. Singh S, Allen AM, Wang Z, et al. Fibrosis Progression in Nonalcoholic Fatty Liver Versus Nonalcoholic Steatohepatitis: A Systematic Review and Meta-Analysis of Paired-Biopsy Studies. Gastroenterology 2014;146:S947-S948.

24. Vilar-Gomez E, Martinez-Perez Y, Calzadilla-Bertot L, et al. Weight Loss Through Lifestyle Modification Significantly Reduces Features of Nonalcoholic Steatohepatitis. Gastroenterology 2015;149:367-+.

25. Loomba R, Abraham M, Unalp A, et al. Association between diabetes, family history of diabetes, and risk of nonalcoholic steatohepatitis and fibrosis. Hepatology 2012;56:943-951.

26. Pelusi S, Cespiati A, Rametta R, et al. Prevalence and Risk Factors of Significant Fibrosis in Patients With Nonalcoholic Fatty Liver Without Steatohepatitis. Clinical Gastroenterology and Hepatology 2019.

27. Han MAT, Altayar O, Hamdeh S, et al. Rates of and Factors Associated With Placebo Response in Trials of Pharmacotherapies for Nonalcoholic Steatohepatitis: Systematic Review and Metaanalysis. Clinical Gastroenterology and Hepatology 2019;17:616-+.

28. McPherson S, Hardy $T$, Henderson E, et al. Evidence of NAFLD progression from steatosis to fibrosing-steatohepatitis using paired biopsies: Implications for prognosis and clinical management. J Hepatol 2015;62:1148-55.

29. Nobili V, Alisi A, Valenti L, et al. NAFLD in children: new genes, new diagnostic modalities and new drugs. Nat Rev Gastroenterol Hepatol 2019.

30. Mardinoglu A, Uhlen M, Boren J. Broad Views of Non-alcoholic Fatty Liver Disease. Cell Systems 2018;6:7-9.

31. Pirola CJ, Sookoian S. Multiomics biomarkers for the prediction of nonalcoholic fatty liver disease severity. World Journal of Gastroenterology 2018;24:1601-1615.

32. Ong JP, Pitts A, Younossi ZM. Increased overall mortality and liver-related mortality in nonalcoholic fatty liver disease. Journal of Hepatology 2008;49:608-612.

33. Frith J, Day CP, Henderson E, et al. Non-Alcoholic Fatty Liver Disease in Older People. Gerontology 2009;55:607-613.

34. Frith J, Jones D, Newton JL. Chronic liver disease in an ageing population. Age and Ageing 2009;38:11-18.

35. Donati B, Valenti L. Telomeres, NAFLD and Chronic Liver Disease. International Journal of Molecular Sciences 2016;17.

36. Papatheodoridi AM, Chrysavgis L, Koutsilieris M, et al. The role of senescence in the development of non-alcoholic fatty liver disease and progression to non-alcoholic steatohepatitis. Hepatology 2019.

37. Kuk JL, Saunders TJ, Davidson LE, et al. Age-related changes in total and regional fat distribution. Ageing Research Reviews 2009;8:339-348.

38. Churilla JR, Fitzhugh EC, Thompson DL. The metabolic syndrome: how definition impacts the prevalence and risk in US adults: 1999-2004 NHANES. Metabolic syndrome and related disorders 2007;5:331-342.

39. Lee YH, Jung KS, Kim SU, et al. Sarcopaenia is associated with NAFLD independently of obesity and insulin resistance: Nationwide surveys (KNHANES 2008-2011). Journal of Hepatology 2015;63:486-493.

40. Lee YH, Kim SU, Song K, et al. Sarcopenia Is Associated With Significant Liver Fibrosis Independently of Obesity and Insulin Resistance in Nonalcoholic Fatty Liver Disease: Nationwide Surveys (KNHANES 2008-2011). Hepatology 2016;63:776-786.

41. Lonardo A, Nascimbeni F, Ballestri S, et al. Sex Differences in NAFLD: State of the Art and Identification of Research Gaps. Hepatology 2019. 
42. Anderson EL, Howe LD, Jones HE, et al. The Prevalence of Non-Alcoholic Fatty Liver Disease in Children and Adolescents: A Systematic Review and Meta-Analysis. PLoS One 2015;10:e0140908.

43. Clark JM, Brancati FL, Diehl AM. Nonalcoholic fatty liver disease. Gastroenterology 2002;122:1649-57.

44. Yang JD, Abdelmalek MF, Pang H, et al. Gender and Menopause Impact Severity of Fibrosis Among Patients With Nonalcoholic Steatohepatitis. Hepatology 2014;59:1406-1414.

45. Yang JD, Suzuki A. The Influence of Menopause on the Development of Hepatic Fibrosis in Nonobese Women With Nonalcoholic Fatty Liver Disease Reply. Hepatology 2014;60:17921793.

46. Klair JS, Yang JD, Abdelmalek MF, et al. A Longer Duration of Estrogen Deficiency Increases Fibrosis Risk Among Postmenopausal Women With Nonalcoholic Fatty Liver Disease. Hepatology 2016;64:85-91.

47. Norheim F, Hui ST, Kulahcioglu E, et al. Genetic and hormonal control of hepatic steatosis in female and male mice. Journal of Lipid Research 2017;58:178-187.

48. Ganz M, Csak T, Szabo G. High fat diet feeding results in gender specific steatohepatitis and inflammasome activation. World Journal of Gastroenterology 2014;20:8525-8534.

49. Lovejoy JC, Champagne CM, de Jonge L, et al. Increased visceral fat and decreased energy expenditure during the menopausal transition. International Journal of Obesity 2008;32:949958.

50. Kurt Z, Barrere-Cain R, LaGuardia J, et al. Tissue-specific pathways and networks underlying sexual dimorphism in non-alcoholic fatty liver disease. Biology of sex differences 2018;9:46.

51. Tomas TC, Urlep Z, Moskon M, et al. LiverSex Computational Model: Sexual Aspects in Hepatic Metabolism and Abnormalities. Frontiers in Physiology 2018;9.

52. Marin V, Rosso N, Dal Ben M, et al. An Animal Model for the Juvenile Non-Alcoholic Fatty Liver Disease and Non-Alcoholic Steatohepatitis. Plos One 2016;11.

53. Rich NE, Oji S, Mufti AR, et al. Racial and Ethnic Disparities in Nonalcoholic Fatty Liver Disease Prevalence, Severity, and Outcomes in the United States: A Systematic Review and Metaanalysis. Clinical Gastroenterology and Hepatology 2018;16:198-+.

54. Zhou F, Zhou J, Wang W, et al. Unexpected Rapid Increase in the Burden of Nonalcoholic Fatty Liver Disease in China From 2008 to 2018: A Systematic Review and Meta-Analysis. Hepatology 2019.

55. Weston SR, Leyden W, Murphy R, et al. Racial and ethnic distribution of nonalcoholic fatty liver in persons with newly diagnosed chronic liver disease. Hepatology 2005;41:372-379.

56. Mohanty SR, Troy TN, Huo D, et al. Influence of ethnicity on histological differences in nonalcoholic fatty liver disease. J Hepatol 2009;50:797-804.

57. Tabibian JH, Lazo M, Durazo FA, et al. Nonalcoholic fatty liver disease across ethno-racial groups: do Asian-American adults represent a new at-risk population? J Gastroenterol Hepatol 2011;26:501-9.

58. Wong VWS, Chu WCW, Wong GLH, et al. Prevalence of non-alcoholic fatty liver disease and advanced fibrosis in Hong Kong Chinese: a population study using proton-magnetic resonance spectroscopy and transient elastography. Gut 2012;61:409-415.

59. Browning JD, Szczepaniak LS, Dobbins R, et al. Prevalence of hepatic steatosis in an urban population in the United States: Impact of ethnicity. Hepatology 2004;40:1387-1395.

60. Guerrero R, Vega GL, Grundy SM, et al. Ethnic differences in hepatic steatosis: an insulin resistance paradox? Hepatology 2009;49:791-801.

61. Romeo S, Kozlitina J, Xing C, et al. Genetic variation in PNPLA3 confers susceptibility to nonalcoholic fatty liver disease. Nat Genet 2008;40:1461-5.

62. Shen J, Wong GLH, Chan HLY, et al. PNPLA3 gene polymorphism accounts for fatty liver in community subjects without metabolic syndrome. Alimentary Pharmacology \& Therapeutics 2014;39:532-539. 
63. Guthold R, Stevens GA, Riley LM, et al. Worldwide trends in insufficient physical activity from 2001 to 2016: a pooled analysis of 358 population-based surveys with 1.9 million participants. Lancet Glob Health 2018;6:e1077-e1086.

64. Bambha $K$, Belt $P, A$ braham $M$, et al. Ethnicity and nonalcoholic fatty liver disease. Hepatology 2012;55:769-780.

65. Chalasani N, Younossi Z, Lavine JE, et al. The diagnosis and management of nonalcoholic fatty liver disease: Practice guidance from the American Association for the Study of Liver Diseases. Hepatology 2018;67:328-357.

66. Saad L. Majority in US drink alcohol, averaging four drinks a week. Gallup Well-Being 2012.

67. Dunn W, Sanyal AJ, Brunt EM, et al. Modest alcohol consumption is associated with decreased prevalence of steatohepatitis in patients with non-alcoholic fatty liver disease (NAFLD). Journal of Hepatology 2012;57:384-391.

68. Kwon HK, Greenson JK, Conjeevaram HS. Effect of lifetime alcohol consumption on the histological severity of non-alcoholic fatty liver disease. Liver International 2014;34:129-135.

69. Ekstedt $\mathrm{M}$, Franzen LE, Holmqvist $\mathrm{M}$, et al. Alcohol consumption is associated with progression of hepatic fibrosis in non-alcoholic fatty liver disease. Scandinavian Journal of Gastroenterology 2009;44:366-374.

70. Ascha MS, Hanouneh IA, Lopez R, et al. The Incidence and Risk Factors of Hepatocellular Carcinoma in Patients with Nonalcoholic Steatohepatitis. Hepatology 2010;51:1972-1978.

71. Meltzer-Brody S, Colquhoun H, Riesenberg R. GBD 2016 Alcohol Collaborators. Alcohol use and burden for 195 countries and territories, 1990-2016: a systematic analysis for the Global Burden of Disease Study 2016 (vol 392, pg 1015, 2018). Lancet 2018;392:1116-1116.

72. Kwon HK, Greenson JK, Conjeevaram HS. Effect of lifetime alcohol consumption on the histological severity of non-alcoholic fatty liver disease. Liver Int 2014;34:129-35.

73. VanWagner LB, Ning HY, Allen NB, et al. Alcohol Use and Cardiovascular Disease Risk in Patients With Nonalcoholic Fatty Liver Disease. Gastroenterology 2017;153:1260-+.

74. Boyle M, Masson S, Anstee QM. The bidirectional impacts of alcohol consumption and the metabolic syndrome: Cofactors for progressive fatty liver disease. J Hepatol 2018;68:251-267.

75. Aberg F, Helenius-Hietala J, Puukka $\mathrm{P}$, et al. Interaction between alcohol consumption and metabolic syndrome in predicting severe liver disease in the general population. Hepatology 2018;67:2141-2149.

76. Chang Y, Cho YK, Kim Y, et al. Nonheavy Drinking and Worsening of Noninvasive Fibrosis Markers in Nonalcoholic Fatty Liver Disease: A Cohort Study. Hepatology 2019;69:64-75.

77. Younossi ZM, Stepanova M, Ong J, et al. Effects of Alcohol Consumption and Metabolic Syndrome on Mortality in Patients with Non-alcoholic and Alcohol-Related Fatty Liver Disease. Clin Gastroenterol Hepatol 2018.

78. Åberg F, Puukka P, Salomaa V, et al. Risks of light and moderate alcohol use in fatty liver disease-follow-up of population cohorts. Hepatology 2019.

79. Ajmera V, Belt P, Wilson LA, et al. Among Patients With Nonalcoholic Fatty Liver Disease, Modest Alcohol Use Is Associated With Less Improvement in Histologic Steatosis and Steatohepatitis. Clinical Gastroenterology and Hepatology 2018;16:1511-+.

80. Lackner C, Tiniakos D. Fibrosis and alcohol-related liver disease. J Hepatol 2019;70:294-304.

81. Rehm J, Taylor B, Mohapatra S, et al. Alcohol as a risk factor for liver cirrhosis: A systematic review and meta-analysis. Drug and Alcohol Review 2010;29:437-445.

82. Romero-Gomez M, Zelber-Sagi S, Trenell M. Treatment of NAFLD with diet, physical activity and exercise. J Hepatol 2017;67:829-846.

83. Trovato FM, Catalano D, Martines GF, et al. Mediterranean diet and non-alcoholic fatty liver disease. The need of extended and comprehensive interventions. Clinical Nutrition 2015;34:86-88. 
84. Estruch R, Ros E, Salas-Salvado J, et al. Primary Prevention of Cardiovascular Disease with a Mediterranean Diet Supplemented with Extra-Virgin Olive Oil or Nuts. N Engl J Med 2018;378:e34.

85. David LA, Maurice CF, Carmody RN, et al. Diet rapidly and reproducibly alters the human gut microbiome. Nature 2014;505:559-+.

86. Leung C, Rivera L, Furness JB, et al. The role of the gut microbiota in NAFLD. Nature Reviews Gastroenterology \& Hepatology 2016;13:412-425.

87. Hoyles L, Fernandez-Real JM, Federici M, et al. Molecular phenomics and metagenomics of hepatic steatosis in non-diabetic obese women. Nature Medicine 2018;24:1070-+.

88. Loomba R, Seguritan V, Li W, et al. Gut Microbiome-Based Metagenomic Signature for Noninvasive Detection of Advanced Fibrosis in Human Nonalcoholic Fatty Liver Disease. Cell Metab 2017;25:1054-1062 e5.

89. Caussy C, Tripathi A, Humphrey G, et al. A gut microbiome signature for cirrhosis due to nonalcoholic fatty liver disease. Nature Communications 2019;10:1406.

90. Caussy C, Hsu C, Lo MT, et al. Link between gut-microbiome derived metabolite and shared gene-effects with hepatic steatosis and fibrosis in NAFLD. Hepatology 2018.

91. Caussy C, Loomba R. Gut microbiome, microbial metabolites and the development of NAFLD. Nat Rev Gastroenterol Hepatol 2018;15:719-720.

92. Arab JP, Karpen SJ, Dawson PA, et al. Bile acids and nonalcoholic fatty liver disease: Molecular insights and therapeutic perspectives. Hepatology 2017;65:350-362.

93. Ilan Y. Leaky gut and the liver: a role for bacterial translocation in nonalcoholic steatohepatitis. World J Gastroenterol 2012;18:2609-18.

94. Enomoto N, Ikejima K, Yamashina S, et al. Kupffer cell sensitization by alcohol involves increased permeability to gut-derived endotoxin. Alcohol Clin Exp Res 2001;25:51S-4S.

95. Miura K, Yang L, van Rooijen N, et al. Toll-like receptor 2 and palmitic acid cooperatively contribute to the development of nonalcoholic steatohepatitis through inflammasome activation in mice. Hepatology 2013;57:577-89.

96. Kawai T, Akira S. TLR signaling. Semin Immunol 2007;19:24-32.

97. Jialal I, Kaur H, Devaraj S. Toll-like receptor status in obesity and metabolic syndrome: a translational perspective. J Clin Endocrinol Metab 2014;99:39-48.

98. Wang J, Thingholm LB, Skieceviciene J, et al. Genome-wide association analysis identifies variation in vitamin $D$ receptor and other host factors influencing the gut microbiota. Nature Genetics 2016;48:1396-1406.

99. Bonder MJ, Kurilshikov A, Tigchelaar EF, et al. The effect of host genetics on the gut microbiome. Nat Genet 2016;48:1407-1412.

100. Hall AB, Tolonen AC, Xavier RJ. Human genetic variation and the gut microbiome in disease. Nature Reviews Genetics 2017;18.

101. Brooks AW, Priya S, Blekhman R, et al. Gut microbiota diversity across ethnicities in the United States. Plos Biology 2018;16.

102. Despres JP. Body Fat Distribution and Risk of Cardiovascular Disease An Update. Circulation 2012;126:1301-1313.

103. Loos RJF, Kilpelainen TO. Genes that make you fat, but keep you healthy. Journal of Internal Medicine 2018;284:450-463.

104. Chitturi S, George J. Interaction of iron, insulin resistance, and nonalcoholic steatohepatitis. Curr Gastroenterol Rep 2003;5:18-25.

105. Eckel N, Li Y, Kuxhaus O, et al. Transition from metabolic healthy to unhealthy phenotypes and association with cardiovascular disease risk across BMI categories in 90257 women (the Nurses' Health Study): 30 year follow-up from a prospective cohort study. Lancet Diabetes Endocrinol 2018;6:714-724. 
106. Caleyachetty R, Thomas GN, Toulis KA, et al. Metabolically Healthy Obese and Incident Cardiovascular Disease Events Among 3.5 Million Men and Women. Journal of the American College of Cardiology 2017;70:1429-1437.

107. Lassale C, Tzoulaki I, Moons KGM, et al. Separate and combined associations of obesity and metabolic health with coronary heart disease: a pan-European case-cohort analysis. Eur Heart J 2018;39:397-406.

108. Winkler TW, Gunther F, Hollerer S, et al. A joint view on genetic variants for adiposity differentiates subtypes with distinct metabolic implications (vol 9, 2018). Nature Communications 2018;9.

109. van der Poorten D, Milner KL, Hui J, et al. Visceral fat: A key mediator of steatohepatitis in metabolic liver disease. Hepatology 2008;48:449-457.

110. Perseghin G. Lipids in the wrong place: visceral fat and nonalcoholic steatohepatitis. Diabetes Care 2011;34 Suppl 2:S367-70.

111. Agbim U, Carr RM, Pickett-Blakely O, et al. Ethnic Disparities in Adiposity: Focus on Nonalcoholic Fatty Liver Disease, Visceral, and Generalized Obesity. Current Obesity Reports 2019;8:243-254.

112. Lim U, Ernst T, Buchthal SD, et al. Asian women have greater abdominal and visceral adiposity than Caucasian women with similar body mass index. Nutrition \& Diabetes 2011;1.

113. Araneta MR, Barrett-Connor E. Ethnic differences in visceral adipose tissue and type 2 diabetes: Filipino, African-American, and white women. Obes Res 2005;13:1458-65.

114. Lear SA, Humphries $\mathrm{KH}$, Kohli $\mathrm{S}$, et al. The use of $\mathrm{BMI}$ and waist circumference as surrogates of body fat differs by ethnicity. Obesity 2007;15:2817-2824.

115. Park YW, Allison DB, Heymsfield SB, et al. Larger amounts of visceral adipose tissue in Asian Americans. Obes Res 2001;9:381-7.

116. Chandalia M, Lin P, Seenivasan T, et al. Insulin resistance and body fat distribution in South Asian men compared to Caucasian men. PLoS One 2007;2:e812.

117. Petersen KF, Dufour S, Feng J, et al. Increased prevalence of insulin resistance and nonalcoholic fatty liver disease in Asian-Indian men. Proc Natl Acad Sci U S A 2006;103:182737.

118. Gujral UP, Pradeepa R, Weber MB, et al. Type 2 diabetes in South Asians: similarities and differences with white Caucasian and other populations. Ann N Y Acad Sci 2013;1281:51-63.

119. Shungin D, Winkler TW, Croteau-Chonka DC, et al. New genetic loci link adipose and insulin biology to body fat distribution. Nature 2015;518:187-U378.

120. Fehlert $\mathrm{E}$, Wagner $\mathrm{R}$, Ketterer $\mathrm{C}$, et al. Genetic determination of body fat distribution and the attributive influence on metabolism. Obesity 2017;25:1277-1283.

121. Lotta LA, Gulati P, Day FR, et al. Integrative genomic analysis implicates limited peripheral adipose storage capacity in the pathogenesis of human insulin resistance ( $\mathrm{vol} 49, \mathrm{pg} 17,2016)$. Nature Genetics 2017;49:317-317.

122. Ji Y, Yiorkas AM, Frau F, et al. Genome-Wide and Abdominal MRI-Imaging Data Provides Evidence that a Genetically Determined Favourable Adiposity Phenotype is Characterized by Lower Ectopic Liver Fat and Lower Risk of Type 2 Diabetes, Heart Disease and Hypertension. Diabetes 2018.

123. Yaghootkar H, Lotta L, Tyrrell J, et al. Genetic Evidence for a Link between Favorable Adiposity and Lower Risk of Type 2 Diabetes, Hypertension, and Heart Disease. Diabetes 2016;65:A421A421.

124. Luukkonen PK, Zhou Y, Sädevirta S, et al. Hepatic ceramides dissociate steatosis and insulin resistance in patients with non-alcoholic fatty liver disease. Journal of hepatology 2016;64:1167-1175.

125. Alonso C, Fernandez-Ramos D, Varela-Rey M, et al. Metabolomic Identification of Subtypes of Nonalcoholic Steatohepatitis. Gastroenterology 2017;152:1449-+. 
126. Scupakova K, Soons Z, Ertaylan G, et al. Spatial Systems Lipidomics Reveals Nonalcoholic Fatty Liver Disease Heterogeneity. Analytical Chemistry 2018;90:5130-5138.

127. Hoang S, Oseini AM, Vincent RK, et al. Development and Validation of a GENE-LEVEL Molecular Disease Activity and Fibrosis Score for Nonalcoholic Fatty LIVER Disease, In HEPATOLOGY, WILEY 111 RIVER ST, HOBOKEN 07030-5774, NJ USA, 2018.

128. Das K, Chowdhury A. Lean NASH: distinctiveness and clinical implication. Hepatol Int 2013;7 Suppl 2:806-13.

129. Ding C, Chan ZL, Magkos F. Lean, but not healthy: the "metabolically obese, normal-weight' phenotype. Current Opinion in Clinical Nutrition and Metabolic Care 2016;19:408-417.

130. Dela Cruz AC, Bugianesi E, George J, et al. Characteristics and Long-Term Prognosis of Lean Patients With Nonalcoholic Fatty Liver Disease. Gastroenterology 2014;146:S909-S909.

131. Hagstrom $\mathrm{H}$, Nasr $\mathrm{P}$, Ekstedt $\mathrm{M}$, et al. Risk for development of severe liver disease in lean patients with nonalcoholic fatty liver disease: A long-term follow-up study. Hepatol Commun 2018;2:48-57.

132. Leung JCF, Loong TCW, Wei JL, et al. Histological Severity and Clinical Outcomes of Nonalcoholic Fatty Liver Disease in Nonobese Patients. Hepatology 2017;65:54-64.

133. Wei JL, Leung JCF, Loong TCW, et al. Prevalence and Severity of Nonalcoholic Fatty Liver Disease in Non-Obese Patients: A Population Study Using Proton-Magnetic Resonance Spectroscopy. American Journal of Gastroenterology 2015;110:1306-1314.

134. Chen F, Esmaili S, Rogers G, et al. Lean NAFLD: A Distinct Entity Shaped by Differential Metabolic Adaptation. Hepatology 2019.

135. Fracanzani AL, Petta S, Lombardi R, et al. Liver and Cardiovascular Damage in Patients With Lean Nonalcoholic Fatty Liver Disease, and Association With Visceral Obesity. Clinical Gastroenterology and Hepatology 2017;15:1604-+.

136. Loomba R, Schork N, Chen CH, et al. Heritability of Hepatic Fibrosis and Steatosis Based on a Prospective Twin Study. Gastroenterology 2015;149:1784-93.

137. Struben VM, Hespenheide EE, Caldwell SH. Nonalcoholic steatohepatitis and cryptogenic cirrhosis within kindreds. Am J Med 2000;108:9-13.

138. Caussy C, Soni M, Cui J, et al. Nonalcoholic fatty liver disease with cirrhosis increases familial risk for advanced fibrosis. J Clin Invest 2017;127:2697-2704.

139. Eslam M, George J. Genetic and epigenetic mechanisms of NASH. Hepatol Int 2016;10:394406.

140. Eslam M, Valenti L, Romeo S. Genetics and epigenetics of NAFLD and NASH: Clinical impact. Journal of Hepatology 2018;68:268-279.

141. Mohlenberg M, Terczynska-Dyla E, Thomsen KL, et al. The role of IFN in the development of NAFLD and NASH. Cytokine 2018.

142. Eslam M, Hashem AM, Leung R, et al. Interferon-lambda rs12979860 genotype and liver fibrosis in viral and non-viral chronic liver disease. Nature Communications 2015;6.

143. Eslam M, McLeod D, Kelaeng KS, et al. IFN-lambda 3, not IFN-lambda 4, likely mediates IFNL3IFNL4 haplotype-dependent hepatic inflammation and fibrosis. Nature Genetics 2017;49:795+ .

144. Metwally M, Bayoumi A, Romero-Gomez M, et al. A polymorphism in the Irisin-encoding gene (FNDC5) associates with hepatic steatosis by differential miRNA binding to the 3'UTR. J Hepatol 2019;70:494-500.

145. Sookoian S, Pirola Cl. Genetics of Nonalcoholic Fatty Liver Disease: From Pathogenesis to Therapeutics. Seminars in Liver Disease 2019;39:124-140.

146. Sookoian S, Pirola CJ. Genetic predisposition in nonalcoholic fatty liver disease. Clinical and Molecular Hepatology 2017;23:1-12.

147. Pelusi S, Baselli G, Pietrelli A, et al. Rare Pathogenic Variants Predispose to Hepatocellular Carcinoma in Nonalcoholic Fatty Liver Disease. Scientific Reports 2019;9. 
148. Nobili V, Liccardo D, Bedogni G, et al. Influence of dietary pattern, physical activity, and I148M PNPLA3 on steatosis severity in at-risk adolescents. Genes Nutr 2014;9:392.

149. Davis JN, Le KA, Walker RW, et al. Increased hepatic fat in overweight Hispanic youth influenced by interaction between genetic variation in PNPLA3 and high dietary carbohydrate and sugar consumption. American Journal of Clinical Nutrition 2010;92:1522-1527.

150. Santoro N, Savoye M, Kim G, et al. Hepatic Fat Accumulation Is Modulated by the Interaction between the rs738409 Variant in the PNPLA3 Gene and the Dietary Omega6/Omega3 PUFA Intake. Plos One 2012;7.

151. Barata L, Feitosa MF, Bielak LF, et al. Insulin Resistance Exacerbates Genetic Predisposition to Nonalcoholic Fatty Liver Disease in Individuals Without Diabetes. Hepatology Communications 2019;3:894-907.

152. Holmen OL, Zhang $\mathrm{H}$, Fan $\mathrm{YB}$, et al. Systematic evaluation of coding variation identifies a candidate causal variant in TM6SF2 influencing total cholesterol and myocardial infarction risk. Nature Genetics 2014;46:345-+.

153. Kozlitina J, Smagris E, Stender S, et al. Exome-wide association study identifies a TM6SF2 variant that confers susceptibility to nonalcoholic fatty liver disease. Nature Genetics 2014;46:352-+.

154. Liu YL, Reeves HL, Burt AD, et al. TM6SF2 rs58542926 influences hepatic fibrosis progression in patients with non-alcoholic fatty liver disease. Nat Commun 2014;5:4309.

155. Eslam M, Mangia A, Berg T, et al. Diverse Impacts of the rs58542926 E167K Variant in TM6SF2 on Viral and Metabolic Liver Disease Phenotypes. Hepatology 2016;64:34-46.

156. Speliotes EK, Yerges-Armstrong LM, Wu J, et al. Genome-Wide Association Analysis Identifies Variants Associated with Nonalcoholic Fatty Liver Disease That Have Distinct Effects on Metabolic Traits. Plos Genetics 2011;7.

157. Liu DJ, Peloso GM, Yu H, et al. Exome-wide association study of plasma lipids in $>300,000$ individuals. Nature Genetics 2017;49:1758-+.

158. Diogo D, Tian C, Franklin CS, et al. Phenome-wide association studies across large population cohorts support drug target validation. Nature Communications 2018;9.

159. Mahajan A, Wessel J, Willems SM, et al. Refining the accuracy of validated target identification through coding variant fine-mapping in type 2 diabetes. Nature Genetics 2018;50:559-+.

160. Abul-Husn NS, Cheng X, Li AH, et al. A Protein-Truncating HSD17B13 Variant and Protection from Chronic Liver Disease. N Engl J Med 2018;378:1096-1106.

161. Mancina RM, Dongiovanni P, Petta S, et al. The MBOAT7-TMC4 Variant rs641738 Increases Risk of Nonalcoholic Fatty Liver Disease in Individuals of European Descent. Gastroenterology 2016;150:1219-1230 e6.

162. Simons N, Isaacs A, Koek GH, et al. PNPLA3, TM6SF2, and MBOAT7 Genotypes and Coronary Artery Disease. Gastroenterology 2017;152:912-913.

163. Thabet K, Asimakopoulos A, Shojaei M, et al. MBOAT7 rs641738 increases risk of liver inflammation and transition to fibrosis in chronic hepatitis C. Nat Commun 2016;7:12757.

164. Liu CH, Ampuero J, Gil-Gomez A, et al. miRNAs in patients with non-alcoholic fatty liver disease: A systematic review and meta-analysis. J Hepatol 2018.

165. Pirola CJ, Gianotti TF, Castano GO, et al. Circulating microRNA signature in non-alcoholic fatty liver disease: from serum non-coding RNAs to liver histology and disease pathogenesis. Gut 2015;64:800-812.

166. Atanasovska B, Rensen SS, van der Sijde MR, et al. A Liver-Specific Long Noncoding RNA With a Role in Cell Viability Is Elevated in Human Nonalcoholic Steatohepatitis. Hepatology 2017;66:794-808.

167. Zhao XY, Xiong XL, Liu TY, et al. Long noncoding RNA licensing of obesity-linked hepatic lipogenesis and NAFLD pathogenesis. Nature Communications 2018;9.

168. Sookoian S, Flichman D, Garaycoechea ME, et al. Metastasis-Associated Lung Adenocarcinoma Transcript 1 as a Common Molecular Driver in the Pathogenesis of Nonalcoholic 
Steatohepatitis and Chronic Immune Mediated Liver Damage. Hepatology Communications 2018;2:654-665.

169. Sookoian S, Rohr C, Salatino A, et al. Genetic variation in long noncoding RNAs and the risk of nonalcoholic fatty liver disease. Oncotarget 2017;8:22917-22926.

170. Ahrens M, Ammerpohl O, von Schonfels W, et al. DNA Methylation Analysis in Nonalcoholic Fatty Liver Disease Suggests Distinct Disease-Specific and Remodeling Signatures after Bariatric Surgery. Cell Metabolism 2013;18:296-302.

171. Murphy SK, Yang HN, Moylan CA, et al. Relationship Between Methylome and Transcriptome in Patients With Nonalcoholic Fatty Liver Disease. Gastroenterology 2013;145:1076-1087.

172. Ma J, Nano J, Ding J, et al. A Peripheral Blood DNA Methylation Signature of Hepatic Fat Reveals a Potential Causal Pathway for Non-Alcoholic Fatty Liver Disease. Diabetes 2019.

173. Hardy $T$, Zeybel M, Day CP, et al. Plasma DNA methylation: a potential biomarker for stratification of liver fibrosis in non-alcoholic fatty liver disease. Gut 2017;66:1321-1328.

174. Kitamoto $T$, Kitamoto $A$, Ogawa $Y$, et al. Targeted-bisulfite sequence analysis of the methylation of CpG islands in genes encoding PNPLA3, SAMM50, and PARVB of patients with non-alcoholic fatty liver disease. J Hepatol 2015;63:494-502.

175. De Chiara F, Heeboll S, Marrone G, et al. Urea cycle dysregulation in non-alcoholic fatty liver disease. Journal of Hepatology 2018;69:905-915.

176. Jalan R, De Chiara F, Balasubramaniyan V, et al. Ammonia produces pathological changes in human hepatic stellate cells and is a target for therapy of portal hypertension. Journal of Hepatology 2016;64:823-833.

177. Thomsen KL, De Chiara F, Rombouts K, et al. Ammonia: A novel target for the treatment of non-alcoholic steatohepatitis. Medical Hypotheses 2018;113:91-97.

178. De Chiara F, Thomsen KL, Habtesion A, et al. Ammonia scavenging prevents progression of fibrosis in experimental non-alcoholic fatty liver disease. Hepatology 2019.

179. Baker PR, 2nd, Friedman JE. Mitochondrial role in the neonatal predisposition to developing nonalcoholic fatty liver disease. J Clin Invest 2018;128:3692-3703.

180. Li Y. Epigenetic Mechanisms Link Maternal Diets and Gut Microbiome to Obesity in the Offspring. Front Genet 2018;9:342.

181. Suter MA, Ma J, Vuguin PM, et al. In utero exposure to a maternal high-fat diet alters the epigenetic histone code in a murine model. Am J Obstet Gynecol 2014;210:463 e1-463 e11.

182. Suter MA, Chen A, Burdine MS, et al. A maternal high-fat diet modulates fetal SIRT1 histone and protein deacetylase activity in nonhuman primates. FASEB J 2012;26:5106-14.

183. Wei YC, Yang CR, Wei YP, et al. Paternally induced transgenerational inheritance of susceptibility to diabetes in mammals. Proceedings of the National Academy of Sciences of the United States of America 2014;111:1873-1878.

184. Aiken CE, Tarry-Adkins JL, Ozanne SE. Transgenerational Developmental Programming of Ovarian Reserve. Scientific Reports 2015;5.

185. Laker RC, Connelly JJ, Yan Z. Exercise Prevents Maternal High-Fat Diet-Induced Hypermethylation of the Pgc-1a Gene and Age-Dependent Metabolic Dysfunction in the Offspring. Diabetes 2014; 63: 160521611. Diabetes 2014;63:E6-E7.

186. Barres R, Zierath JR. The role of diet and exercise in the transgenerational epigenetic landscape of T2DM. Nature Reviews Endocrinology 2016;12:441-451.

187. Ajmera VH, Terrault NA, VanWagner LB, et al. Longer lactation duration is associated with decreased prevalence of non-alcoholic fatty liver disease in women. Journal of Hepatology 2019;70:126-132.

188. Ayonrinde OT, Oddy WH, Adams LA, et al. Infant nutrition and maternal obesity influence the risk of non-alcoholic fatty liver disease in adolescents. J Hepatol 2017;67:568-576.

189. Sookoian S, Rosselli MS, Gemma C, et al. Epigenetic regulation of insulin resistance in nonalcoholic fatty liver disease: impact of liver methylation of the peroxisome proliferatoractivated receptor gamma coactivator 1alpha promoter. Hepatology 2010;52:1992-2000. 
190. Gemma C, Sookoian S, Alvarinas J, et al. Maternal Pregestational BMI Is Associated With Methylation of the PPARGC1A Promoter in Newborns. Obesity 2009;17:1032-1039.

191. McPherson S, Hardy T, Dufour JF, et al. Age as a Confounding Factor for the Accurate NonInvasive Diagnosis of Advanced NAFLD Fibrosis. American Journal of Gastroenterology 2017;112:740-751.

192. De Silva S, Li WH, Kemos P, et al. Non-invasive markers of liver fibrosis in fatty liver disease are unreliable in people of South Asian descent. Frontline Gastroenterology 2018;9:115-121.

193. Santhekadur PK, Kumar DP, Sanyal AJ. Preclinical models of non-alcoholic fatty liver disease. Journal of Hepatology 2018;68:230-237.

194. Tu LN, Showalter MR, Cajka T, et al. Metabolomic characteristics of cholesterol-induced nonobese nonalcoholic fatty liver disease in mice. Scientific Reports 2017;7.

195. Drew L. Development pipeline review 2018. Nature 2017;551:S86-S89.

196. Woodcock J, LaVange LM. Master protocols to study multiple therapies, multiple diseases, or both. New England Journal of Medicine 2017;377:62-70.

197. Bhatt DL, Mehta C. Adaptive Designs for Clinical Trials. N Engl J Med 2016;375:65-74.

198. Brunt EM, Ramrakhiani S, Cordes BG, et al. Concurrence of histologic features of steatohepatitis with other forms of chronic liver disease. Modern Pathology 2003;16:49-56.

199. Sanchez-Munoz D, Castellano-Megias VM, Romero-Gomez M. Histologic features of steatohepatitis in patients with a clinical diagnosis of autoimmune cholestasis. Dig Dis Sci 2004;49:1957-61.

200. Cotrim HP, Andrade ZA, Parana R, et al. Nonalcoholic steatohepatitis: a toxic liver disease in industrial workers. Liver 1999;19:299-304.

201. Choi HS, Brouwer WP, Zanjir WM, et al. Non-Alcoholic steatohepatitis is associated with liverrelated outcomes and all-cause mortality in chronic hepatitis B. Hepatology 2019.

202. Chiang DJ, McCullough AJ. The Impact of Obesity and Metabolic Syndrome on Alcoholic Liver Disease. Clinics in Liver Disease 2014;18:157-+.

203. Beuers U, Gershwin ME, Gish RG, et al. Changing nomenclature for PBC: from 'cirrhosis' to 'cholangitis'. Gastroenterology 2015;149:1627-9. 